(n)

trobertivier

Journal of Nonlinear Mathematical Physics

ISSN (Online): 1776-0852 ISSN (Print): 1402-9251

Journal Home Page: https://www.atlantis-press.com/journals/jnmp

\title{
Periodic orbits associated to Hamiltonian functions of degree four
}

Dante Carrasco-Olivera, Marco Uribe, Claudio Vidal

To cite this article: Dante Carrasco-Olivera, Marco Uribe, Claudio Vidal (2014) Periodic orbits associated to Hamiltonian functions of degree four, Journal of Nonlinear Mathematical Physics 21:3, 336-356, DOI:

https://doi.org/10.1080/14029251.2014.936756

To link to this article: https://doi.org/10.1080/14029251.2014.936756

Published online: 04 January 2021 


\title{
Periodic orbits associated to Hamiltonian functions of degree four
}

\author{
Dante Carrasco-Olivera* \\ Departamento de Matemática, Universidad del Bío- Bío \\ Avda. Collao 1202, Concepcion, Chile \\ dcarrasc@ubiobio.cl
}

Marco Uribe ${ }^{\dagger}$

Facultad de Ingeniería, Departamento de Matemática y Física Aplicadas, Universidad Católica de la Ssma. Concepción Casilla 297, Concepcion, Chile

muribe@ucsc.cl

Claudio Vidal ${ }^{\ddagger}$

Departamento de Matemática, Universidad del Bío- Bío

Avda. Collao 1202, Concepcion, Chile

clvidal@ubiobio.cl

Received 24 January 2014

Accepted 25 March 2014

\begin{abstract}
We consider the Hamiltonian polynomial function $H$ of degree fourth given by either $H\left(x, y, p_{x}, p_{y}\right)=\frac{1}{2}\left(p_{x}^{2}+\right.$ $\left.p_{y}^{2}\right)+\frac{1}{2}\left(x^{2}+y^{2}\right)+V_{3}(x, y)+V_{4}(x, y)$, or $H\left(x, y, p_{x}, p_{y}\right)=\frac{1}{2}\left(-p_{x}^{2}+p_{y}^{2}\right)+\frac{1}{2}\left(-x^{2}+y^{2}\right)+V_{3}(x, y)+V_{4}(x, y)$, where $V_{3}(x, y)$ and $V_{4}(x, y)$ are homogeneous polynomials of degree three and four, respectively. Our main objective is to prove the existence and stability of periodic solutions associated to $H$ using the classical averaging method.
\end{abstract}

Keywords: Hamiltonian systems; Periodic orbits; Stability; Averaging theory.

2000 Mathematics Subject Classification: Primary 34C29, 37J25, 34C25; Secondary 85A05.

\section{Introduction}

It is known that the periodic orbits are the most simple non-trivial solutions of an ordinary differential system, and that their study is of particular interest because the motion in a neighborhood can be determined by their type of stability, [22]. In this paper we study the existence and stability of periodic solutions in Hamiltonian systems defined for two families of polynomial Hamiltonians of degree four on the plane. More specifically, we consider the following polynomial Hamiltonian

\footnotetext{
${ }^{*}$ The first author was partially supported by FONDECYT project 11121598, CONICYT (Chile), Grupo de Investigación Sistemas Dinámicos y Aplicaciones GI120708/EF, UBB (Chile) and Post-Doctorado Verano 2014, IMPA, Rio de Janeiro, Brazil.

†The second author was partially supported by FONDECYT project 11080250, CONICYT (Chile), Post-Doctorado Verano 2014, IMPA, Rio de Janeiro, Brazil and Dirección de Investigación UCSC, Concepción (Chile).

$¥$ The third author was partially supported by FONDECYT project 1130644, CONICYT (Chile) and by Grupo de Investigación Sistemas Dinámicos y Aplicaciones GI120708/EF, UBB (Chile).
} 
functions:

$$
H^{+}=\frac{1}{2}\left(p_{x}^{2}+p_{y}^{2}\right)+\frac{1}{2}\left(x^{2}+y^{2}\right)+V_{3}(x, y)+V_{4}(x, y),
$$

and

$$
H^{-}=\frac{1}{2}\left(-p_{x}^{2}+p_{y}^{2}\right)+\frac{1}{2}\left(-x^{2}+y^{2}\right)+V_{3}(x, y)+V_{4}(x, y),
$$

where the polynomials $V_{3}$ and $V_{4}$ are homogeneous polynomials of the third and fourth degree, respectively and which are given by

$$
V_{3}(x, y)=\frac{A}{3} x^{3}+B x y^{2},
$$

and

$$
V_{4}(x, y)=\frac{\Lambda}{4} x^{4}+\frac{m}{2} x^{2} y^{2}+\frac{\lambda}{4} y^{4},
$$

where $A, B, \Lambda, m, \lambda$ are real parameters.

In this work we will make use of the averaging method to find families of periodic orbits associated to the Hamiltonian given by (1.1) and (1.2), respectively, for appropriate conditions on the parameters $A, B, m, \lambda$ and $\Lambda$. The essential tools that we use are the definitions and notations on the averaging method which are given in [6] (see also [27]). Others studies related to the Hénon-Heiles system can be found in: [1], [2], [4], [5], [8], [9], [10], [12], [13], [14], [15], [18], [19], [23], [26] and references therein. In [7], the authors study the dynamics associated to (1.1) in a much more general sense but the case of the cosmological model (case $\mathrm{H}^{-}$) is not considered.

Before to enunciate the main results in this paper, we introduce the appropriate notations in relation to the parameters of the Hamiltonian (1.1) and (1.2), respectively. For the constants $A, B, m, \lambda, \Lambda$, we define

$$
\begin{aligned}
& M_{1}=10 B(A+B)+9(\lambda-m) \\
& M_{2}=10(A-3 B)(A+B)-9(\lambda+\Lambda-2 m) \\
& M_{3}=10(A-2 B)(A+B)+9(m-\Lambda) \\
& M_{4}=2(A-6 B) B+3 m \\
& M_{5}=14 B(A-B)+3(3 \lambda-m) \\
& M_{6}=2(5 A-9 B)(A-B)-9(\lambda+\Lambda)+6 m \\
& M_{7}=2(5 A-2 B)(A-B)-3(3 \Lambda-m) .
\end{aligned}
$$

Once fixed $h>0$ and the parameters $A, B, m, \lambda, \Lambda$, we consider the following sets:

$$
\Lambda_{h}^{1}=\left\{(A, B, m, \lambda, \Lambda) \in \mathbb{R}^{5}: \frac{M_{1}}{M_{2}}<0, \frac{M_{3}}{M_{2}}>0, M_{3} M_{4} \neq 0\right\}
$$

and

$$
\Lambda_{h}^{2}=\left\{(A, B, m, \lambda, \Lambda) \in \mathbb{R}^{5}: \frac{M_{5}}{M_{6}}<0, \frac{M_{7}}{M_{6}}>0, M_{4} M_{7} \neq 0\right\},
$$

then we get the following result: 
Theorem 1.1. Let $h>0$ and $H$ be as in (1.1). Then:

(1) there is at least one family of periodic orbits if $(A, B, m, \lambda, \Lambda) \in \Lambda_{h}^{1} \backslash \Lambda_{h}^{2}$.

(2) there are at least two families of periodic orbits if $(A, B, m, \lambda, \Lambda) \in \Lambda_{h}^{2} \backslash \Lambda_{h}^{1}$.

(3) there are at least three families of periodic orbits if $(A, B, m, \lambda, \Lambda) \in \Lambda_{h}^{1} \cap \Lambda_{h}^{2}$.

The next theorem gives information on the stability and unstability of the periodic solutions obtained in Theorem 1.1.

Theorem 1.2. Under the same hypotheses of Theorem 1.1 we have that:

(i) The family in (a) is linearly stable if $M_{3} M_{4}>0$ and unstable if $M_{3} M_{4}<0$.

(ii) The two families in (b) are linearly stable if $M_{4} M_{7}<0$ and unstable if $M_{4} M_{7}>0$.

(iii) The three families in (c) are linearly stable if $M_{3} M_{4}>0$ and $M_{4} M_{7}<0$, and unstable if either

(A1) $M_{3} M_{4}>0$ and $M_{4} M_{7}>0$, or

(A2) $M_{3} M_{4}<0$ and $M_{4} M_{7}>0$, or

(A3) $M_{3} M_{4}<0$ and $M_{4} M_{7}<0$.

In a similar way, for $A, B, m, \lambda, \Lambda$, we define the following expressions:

$$
\begin{aligned}
& N_{1}=10 B(A-B)+9(\lambda+m) \\
& N_{2}=10(A-B)(A+3 B)+9(\lambda+\Lambda+2 m) \\
& N_{3}=10(A-B)(A+2 B)+9(\Lambda+m) \\
& N_{4}=2 B(A+6 B)-3 m \\
& N_{5}=14 B(A+B)+3(3 \lambda+m) \\
& N_{6}=2(A+B)(5 A+9 B)+9(\lambda+\Lambda)+6 m \\
& N_{7}=2(A+B)(5 A+2 B)+3(3 \Lambda+m) .
\end{aligned}
$$

Fixed $h \neq 0$ small and the parameters $A, B, m, \lambda, \Lambda$, we consider the following sets:

$$
\Omega_{h}^{1}=\left\{(A, B, m, \lambda, \Lambda) \in \mathbb{R}^{5}:-2 h \frac{N_{1}}{N_{2}}>0,2 h \frac{N_{3}}{N_{2}}>0, N_{3} N_{4} \neq 0\right\}
$$

and

$$
\Omega_{h}^{2}=\left\{(A, B, m, \lambda, \Lambda) \in \mathbb{R}^{5}:-2 h \frac{N_{5}}{N_{6}}>0,2 h \frac{N_{7}}{N_{6}}>0, N_{4} N_{7} \neq 0\right\},
$$

then we get the following result:

Theorem 1.3. For any $h \neq 0$, the Hamiltonian system associated to (1.2) has at least:

(a) one family of period orbits if $(A, B, m, \lambda, \Lambda) \in \Omega_{h}^{1} \backslash \Omega_{h}^{2}$.

(b) two families of period orbits if $(A, B, m, \lambda, \Lambda) \in \Omega_{h}^{2} \backslash \Omega_{h}^{1}$.

(c) three families of period orbits if $(A, B, m, \lambda, \Lambda) \in \Omega_{h}^{1} \cap \Omega_{h}^{2}$.

The next theorem gives us information on the stability or unstability of the periodic orbits given in Theorem 1.3.

Theorem 1.4. Under the same hypotheses of Theorem 1.3 we have that: 
(i) The family in (a) is linearly stable if $h N_{3} N_{4}>0$ and unstable if $h N_{3} N_{4}<0$.

(ii) The two families in (b) are linearly stable if $h N_{4} N_{7}<0$ and unstable if $h N_{4} N_{7}>0$.

(iii) The three families in (c) are linearly stable if $h N_{3} N_{4}>0$ and $h N_{4} N_{7}<0$, and unstable if either
(A1) $h N_{3} N_{4}>0$ and $h N_{4} N_{7}>0$, or
(A2) $h N_{3} N_{4}<0$ and $h N_{4} N_{7}>0$, or
(A3) $h N_{3} N_{4}<0$ and $h N_{4} N_{7}<0$.

Theorems 1.1 and 1.2 are improvement of results given in [18] and Theorems 1.3 and 1.4 are improvement of results given in [1]. The strategy to add more terms to the classical models of cosmological scalar fields in [18] is because such perturbation increases the regions of existence of periodic orbits.

Now, we point out that since our potential $V=V_{3}+V_{4}$ is the addition of the homogeneous polynomials of degree three and four defined in (1.3) and (1.4) they have the property of symmetry, in fact, the reflection $y \rightarrow-y$. Thus, we can apply symmetry arguments, but will not be studied in this work. On the other hand, since the Hamiltonian function $H^{+}$(and $H^{-}$) has the origin $(0,0,0,0)$ as an equilibrium point whose eigenvalues of the linearization are $\pm i$ with multiplicity two and the linear part is diagonalizable and the Hessian evaluated at $(0,0,0,0)$ is positive definite. Then by Weinstein's Theorem (see [22]) we can prove the existence of at least two (families) periodic solutions whose periods are close to $2 \pi$ and the energy level $h=H^{+}$is sufficiently close to 0 . But, we cannot apply this theorem for $H^{-}$.

It is important to observe that the used averaging method is closely reminiscent of the normal form approach: therefore, methods very similar to those exploited by us have been used in many occasions before, especially to find periodic orbits, see for example [3], [11], [20], [21] and [28]. On the other hand, the natural systems with indefinite kinetic energy (the "cosmological Hamiltonian" in the language of our work) have recently been investigated by [24] and [25].

The organization of the paper is as follows: In Sections 2 and 3, we will describe the motion equations to apply the Averaging theory. The main results will be proved in Section 4 and 5. Finally, in Section 6 we will give examples where the conditions of the main results are not empty.

\section{Statement of the problem and equations of motion for $H^{+}$}

The equations of motion associated to the system (1.1) and are

$$
\begin{aligned}
\dot{x} & =p_{x}, \\
\dot{y} & =p_{y}, \\
\dot{p}_{x} & =-x-\frac{\partial V_{3}}{\partial x}-\frac{\partial V_{4}}{\partial x}, \\
\dot{p}_{y} & =-y-\frac{\partial V_{3}}{\partial y}-\frac{\partial V_{4}}{\partial y} .
\end{aligned}
$$


Let $x=\sqrt{\varepsilon} X, y=\sqrt{\varepsilon} Y, p_{x}=\sqrt{\varepsilon} p_{X}$ and $p_{y}=\sqrt{\varepsilon} p_{Y}$ be the change of variable, which is $\varepsilon^{-2}$ symplectic, therefore the system (2.1) becomes

$$
\begin{aligned}
\dot{X} & =p_{X}, \\
\dot{Y} & =p_{Y}, \\
\dot{p}_{X} & =-X-\varepsilon \frac{\partial V_{3}}{\partial X}-\varepsilon^{2} \frac{\partial V_{4}}{\partial X}, \\
\dot{p}_{Y} & =-Y-\varepsilon \frac{\partial V_{3}}{\partial Y}-\varepsilon^{2} \frac{\partial V_{4}}{\partial Y} .
\end{aligned}
$$

The Hamiltonian function associated to (2.1) and (2.2) is

$$
K^{+}=\frac{1}{2}\left(p_{X}^{2}+p_{Y}^{2}\right)+\frac{1}{2}\left(X^{2}+Y^{2}\right)+\varepsilon V_{3}+\varepsilon^{2} V_{4}
$$

where $K^{+}=\varepsilon^{-2} H^{+}=h$.

By the standard theory of Hamiltonian dynamical systems, for all $\varepsilon$ different from zero, the original system (2.1) and the new system (2.2) have essentially the same phase portrait, and additionally the system (2.2), for $\varepsilon$ sufficiently small, is close to an integrable system.

Now, we introduce the change of variables $(r, \rho, \theta, \alpha)$ by the relations

$$
X=r \cos \theta, \quad Y=\rho \cos (\theta+\alpha), \quad p_{X}=r \sin \theta, \quad p_{Y}=\rho \sin (\theta+\alpha) .
$$

Recall that this is a well defined change of variables when $r>0$ and $\rho>0$. Clearly this change of variables is not canonical, so we lose the Hamiltonian structure of the system of differential equations. Next, differentiating directly and using the expressions given in (2.2) we obtain

$$
\begin{aligned}
& \dot{r}=-\varepsilon \sin \theta \frac{\partial V_{3}}{\partial X}-\varepsilon^{2} \sin \theta \frac{\partial V_{4}}{\partial X}, \\
& \dot{\theta}=-1-\varepsilon \frac{\cos \theta}{r} \frac{\partial V_{3}}{\partial X}-\varepsilon^{2} \frac{\cos \theta}{r} \frac{\partial V_{4}}{\partial X}, \\
& \dot{\rho}=-\varepsilon \sin (\theta+\alpha) \frac{\partial V_{3}}{\partial Y}-\varepsilon^{2} \sin (\theta+\alpha) \frac{\partial V_{4}}{\partial Y}, \\
& \dot{\alpha}=\varepsilon\left[\frac{\cos \theta}{r} \frac{\partial V_{3}}{\partial X}-\frac{\cos (\theta+\alpha)}{\rho} \frac{\partial V_{3}}{\partial Y}\right]+\varepsilon^{2}\left[\frac{\cos \theta}{r} \frac{\partial V_{4}}{\partial X}-\frac{\cos (\theta+\alpha)}{\rho} \frac{\partial V_{4}}{\partial Y}\right],
\end{aligned}
$$

where the partial derivatives of $V_{3}$ and $V_{4}$ are evaluated at the point $(r \cos \theta, \rho \cos (\theta+\alpha))$. Moreover note that

$$
\begin{aligned}
& \frac{\partial V_{k}}{\partial r}=\cos \theta \frac{\partial V_{k}}{\partial X} \\
& \frac{\partial V_{k}}{\partial \theta}=-r \sin \theta \frac{\partial V_{k}}{\partial X}-\rho \sin (\theta+\alpha) \frac{\partial V_{k}}{\partial Y} \\
& \frac{\partial V_{k}}{\partial \rho}=\cos (\theta+\alpha) \frac{\partial V_{k}}{\partial Y} \\
& \frac{\partial V_{k}}{\partial \alpha}=-\rho \sin (\theta+\alpha) \frac{\partial V_{k}}{\partial Y} .
\end{aligned}
$$


for $k=3,4$. Therefore (2.4) can be written as

$$
\begin{aligned}
& \dot{r}=\varepsilon \frac{1}{r}\left(\frac{\partial V_{3}}{\partial \theta}-\frac{\partial V_{3}}{\partial \alpha}\right)+\varepsilon^{2} \frac{1}{r}\left(\frac{\partial V_{4}}{\partial \theta}-\frac{\partial V_{4}}{\partial \alpha}\right), \\
& \dot{\theta}=-1-\varepsilon \frac{1}{r} \frac{\partial V_{3}}{\partial r}-\varepsilon^{2} \frac{1}{r} \frac{\partial V_{4}}{\partial r}, \\
& \dot{\rho}=\varepsilon \frac{1}{\rho} \frac{\partial V_{3}}{\partial \alpha}+\varepsilon^{2} \frac{1}{\rho} \frac{\partial V_{4}}{\partial \alpha} \\
& \dot{\alpha}=\varepsilon\left(\frac{1}{r} \frac{\partial V_{3}}{\partial r}-\frac{1}{\rho} \frac{\partial V_{3}}{\partial \rho}\right)+\varepsilon^{2}\left(\frac{1}{r} \frac{\partial V_{4}}{\partial r}-\frac{1}{\rho} \frac{\partial V_{4}}{\partial \rho}\right) .
\end{aligned}
$$

We observe that for a fixed value $h$ of $K^{+}$, in polar coordinates, it assumes the form

$$
h=\frac{1}{2}\left(r^{2}+\rho^{2}\right)+\varepsilon V_{3}(r \cos \theta, \rho \cos (\theta+\alpha))+\varepsilon^{2} V_{4}(r \cos \theta, \rho \cos (\theta+\alpha)) .
$$

In order to put our system as a periodic ordinary differential equation, we introduce the $\theta$ variable as independent (new time), and we use the notation prime to denote the derivative with respect to $\theta$. It is observed that the angular variable $\alpha$ cannot be used as the independent variable, since the new differential system do not have the appropriate form in order to apply the averaging method. Now, note that

$$
\frac{d r}{d \theta}=-\frac{\varepsilon\left(\frac{\partial V_{3}}{\partial \theta}-\frac{\partial V_{3}}{\partial \alpha}\right)+\varepsilon^{2}\left(\frac{\partial V_{4}}{\partial \theta}-\frac{\partial V_{4}}{\partial \alpha}\right)}{r+\varepsilon \frac{\partial V_{3}}{\partial r}+\varepsilon^{2} \frac{\partial V_{4}}{\partial r}}
$$

Remember that the function

$$
f(\varepsilon)=\frac{1}{1+a_{1} \varepsilon+a_{2} \varepsilon^{2}}=1-a_{1} \varepsilon+\left(a_{1}^{2}-a_{2}\right) \varepsilon^{2}+O\left(\varepsilon^{3}\right),
$$

by developing a Taylor series in powers of $\varepsilon$ around $\varepsilon=0$. Replacing $a_{1}=\frac{1}{r} \frac{\partial V_{3}}{\partial r}$ and $a_{2}=\frac{1}{r} \frac{\partial V_{4}}{\partial r}$ we get

$$
\frac{1}{1+\frac{1}{r} \frac{\partial V_{3}}{\partial r} \varepsilon+\frac{1}{r} \frac{\partial V_{4}}{\partial r} \varepsilon^{2}}=1-\frac{1}{r} \frac{\partial V_{3}}{\partial r} \varepsilon+\left[\left(\frac{1}{r} \frac{\partial V_{3}}{\partial r}\right)^{2}-\frac{1}{r} \frac{\partial V_{4}}{\partial r}\right] \varepsilon^{2}+O\left(\varepsilon^{3}\right) .
$$

Therefore,

$$
r^{\prime}=-\frac{1}{r}\left(\frac{\partial V_{3}}{\partial \theta}-\frac{\partial V_{3}}{\partial \alpha}\right) \varepsilon+\frac{1}{r}\left[\frac{1}{r} \frac{\partial V_{3}}{\partial r}\left(\frac{\partial V_{3}}{\partial \theta}-\frac{\partial V_{3}}{\partial \alpha}\right)-\left(\frac{\partial V_{4}}{\partial \theta}-\frac{\partial V_{4}}{\partial \alpha}\right)\right] \varepsilon^{2}+O\left(\varepsilon^{3}\right) .
$$

Moreover,

$$
\frac{d \rho}{d \theta}=-\frac{\varepsilon \frac{1}{\rho} \frac{\partial V_{3}}{\partial \alpha}+\varepsilon^{2} \frac{1}{\rho} \frac{\partial V_{4}}{\partial \alpha}}{1+\varepsilon \frac{1}{r} \frac{\partial V_{3}}{\partial r}+\varepsilon^{2} \frac{1}{r} \frac{\partial V_{4}}{\partial r}}
$$


thus

$$
\rho^{\prime}=-\frac{1}{\rho} \frac{\partial V_{3}}{\partial \alpha} \varepsilon+\left[\frac{1}{r \rho} \frac{\partial V_{3}}{\partial r} \frac{\partial V_{3}}{\partial \alpha}-\frac{1}{\rho} \frac{\partial V_{4}}{\partial \alpha}\right] \varepsilon^{2}+O\left(\varepsilon^{3}\right) .
$$

Also

$$
\frac{d \alpha}{d \theta}=-\frac{\varepsilon\left(\frac{1}{r} \frac{\partial V_{3}}{\partial r}-\frac{1}{\rho} \frac{\partial V_{3}}{\partial \rho}\right)+\varepsilon^{2}\left(\frac{1}{r} \frac{\partial V_{4}}{\partial r}-\frac{1}{\rho} \frac{\partial V_{4}}{\partial \rho}\right)}{1+\varepsilon \frac{1}{r} \frac{\partial V_{3}}{\partial r}+\varepsilon^{2} \frac{1}{r} \frac{\partial V_{4}}{\partial r}},
$$

then

$$
\alpha^{\prime}=-\left(\frac{1}{r} \frac{\partial V_{3}}{\partial r}-\frac{1}{\rho} \frac{\partial V_{3}}{\partial \rho}\right) \varepsilon+\left[\frac{1}{r} \frac{\partial V_{3}}{\partial r}\left(\frac{1}{r} \frac{\partial V_{3}}{\partial r}-\frac{1}{\rho} \frac{\partial V_{3}}{\partial \rho}\right)-\left(\frac{1}{r} \frac{\partial V_{4}}{\partial r}-\frac{1}{\rho} \frac{\partial V_{4}}{\partial \rho}\right)\right] \varepsilon^{2}+O\left(\varepsilon^{3}\right) .
$$

If we write the previous system as a Taylor series in powers of $\varepsilon$, we have that

$$
\begin{aligned}
r^{\prime}= & -\frac{1}{r}\left(\frac{\partial V_{3}}{\partial \theta}-\frac{\partial V_{3}}{\partial \alpha}\right) \varepsilon+\frac{1}{r}\left[\frac{1}{r} \frac{\partial V_{3}}{\partial r}\left(\frac{\partial V_{3}}{\partial \theta}-\frac{\partial V_{3}}{\partial \alpha}\right)-\left(\frac{\partial V_{4}}{\partial \theta}-\frac{\partial V_{4}}{\partial \alpha}\right)\right] \varepsilon^{2}+O\left(\varepsilon^{3}\right) \\
\rho^{\prime}= & -\frac{1}{\rho} \frac{\partial V_{3}}{\partial \alpha} \varepsilon+\left[\frac{1}{r \rho} \frac{\partial V_{3}}{\partial r} \frac{\partial V_{3}}{\partial \alpha}-\frac{1}{\rho} \frac{\partial V_{4}}{\partial \alpha}\right] \varepsilon^{2}+O\left(\varepsilon^{3}\right), \\
\alpha^{\prime}= & -\left(\frac{1}{r} \frac{\partial V_{3}}{\partial r}-\frac{1}{\rho} \frac{\partial V_{3}}{\partial \rho}\right) \varepsilon+\left[\frac{1}{r} \frac{\partial V_{3}}{\partial r}\left(\frac{1}{r} \frac{\partial V_{3}}{\partial r}-\frac{1}{\rho} \frac{\partial V_{3}}{\partial \rho}\right)-\left(\frac{1}{r} \frac{\partial V_{4}}{\partial r}-\frac{1}{\rho} \frac{\partial V_{4}}{\partial \rho}\right)\right] \varepsilon^{2}+ \\
& O\left(\varepsilon^{3}\right)
\end{aligned}
$$

In order to apply the averaging theory we will fix the value of the first integral of $K=h>0$. We write the Taylor expansion of the function $\rho$ of second order in $\varepsilon$ and rewrite the system (2.7) in the variables $r$ and $\alpha$. The function $\rho$ in its Taylor form is

$$
\rho(\varepsilon)=\rho(0)+\rho^{\prime}(0) \varepsilon+O\left(\varepsilon^{2}\right) .
$$

Regarding (2.8), we obtain for $\varepsilon=0$,

$$
\rho(0)=\sqrt{2 h-r^{2}} .
$$

Derivating (2.8) with respect to $\varepsilon$, we have

$$
\begin{aligned}
0= & \rho \rho^{\prime}+V_{3}(r \cos \theta, \rho \cos (\theta+\alpha))+\varepsilon \frac{\partial V_{3}}{\partial Y} \rho^{\prime} \cos (\theta+\alpha)+ \\
& 2 \varepsilon V_{4}(r \cos \theta, \rho \cos (\theta+\alpha))+\varepsilon^{2} \frac{\partial V_{4}}{\partial Y} \rho^{\prime} \cos (\theta+\alpha)
\end{aligned}
$$

and evaluating at $\varepsilon=0$, we obtain

$$
\rho^{\prime}(0)=-\frac{1}{\sqrt{2 h-r^{2}}} V_{3}\left(r \cos \theta, \sqrt{2 h-r^{2}} \cos (\theta+\alpha)\right) .
$$

Thus, from (2.8), (2.9) and (2.10) the development of the function $\rho$ in Taylor series has the form

$$
\rho(\varepsilon)=\sqrt{2 h-r^{2}}-\frac{1}{\sqrt{2 h-r^{2}}} V_{3}\left(r \cos \theta, \sqrt{2 h-r^{2}} \cos (\theta+\alpha)\right) \varepsilon+O\left(\varepsilon^{2}\right) .
$$


Since $V_{3}$ depends on the variables $(r, \rho, \theta, \alpha)$, the derivatives of $V_{3}$ respect to their variables in the Taylor series are:

- $\frac{\partial V_{3}}{\partial \theta}=\frac{\partial V_{3}}{\partial \theta}+\rho^{\prime}(0) \frac{\partial^{2} V_{3}}{\partial \rho \partial \theta} \varepsilon+O\left(\varepsilon^{2}\right)$,

- $\frac{\partial V_{3}}{\partial \alpha}=\frac{\partial V_{3}}{\partial \alpha}+\rho^{\prime}(0) \frac{\partial^{2} V_{3}}{\partial \rho \partial \alpha} \varepsilon+O\left(\varepsilon^{2}\right)$,

- $\frac{\partial V_{3}}{\partial r}=\frac{\partial V_{3}}{\partial r}+\rho^{\prime}(0) \frac{\partial^{2} V_{3}}{\partial \rho \partial r} \varepsilon+O\left(\varepsilon^{2}\right)$,

- $\frac{\partial V_{3}}{\partial \rho}=\frac{\partial V_{3}}{\partial \rho}+\rho^{\prime}(0) \frac{\partial^{2} V_{3}}{\partial \rho^{2}} \varepsilon+O\left(\varepsilon^{2}\right)$

Moreover,

- $\frac{1}{\rho}=\frac{1}{\rho(0)}-\frac{\rho^{\prime}(0)}{\rho^{2}(0)} \varepsilon+O\left(\varepsilon^{2}\right)$,

- $\frac{1}{\rho} \frac{\partial V_{3}}{\partial \rho}=\frac{1}{\rho(0)} \frac{\partial V_{3}}{\partial \rho}+\left[\frac{\rho^{\prime}(0)}{\rho(0)} \frac{\partial^{2} V_{3}}{\partial \rho^{2}}-\frac{\rho^{\prime}(0)}{\rho^{2}(0)} \frac{\partial V_{3}}{\partial \rho}\right] \varepsilon+O\left(\varepsilon^{2}\right)$.

Recall that the function $V_{3}$ and its partial derivatives are evaluated at the point $(r \cos \theta, \rho(0) \cos (\theta+$ $\alpha)$ ), with $\rho(0)$ as in (2.9). Therefore,

$$
\begin{aligned}
\frac{1}{r}\left(\frac{\partial V_{3}}{\partial \theta}-\frac{\partial V_{3}}{\partial \alpha}\right) & =\frac{1}{r}\left(\frac{\partial V_{3}}{\partial \theta}-\frac{\partial V_{3}}{\partial \alpha}\right)+\frac{\rho^{\prime}(0)}{r}\left(\frac{\partial^{2} V_{3}}{\partial \rho \partial \theta}-\frac{\partial^{2} V_{3}}{\partial \rho \partial \alpha}\right) \varepsilon \\
\left(\frac{1}{r} \frac{\partial V_{3}}{\partial r}-\frac{1}{\rho} \frac{\partial V_{3}}{\partial \rho}\right) & =\left(\frac{1}{r} \frac{\partial V_{3}}{\partial r}-\frac{1}{\rho(0)} \frac{\partial V_{3}}{\partial \rho}\right)+\left[\frac{\rho^{\prime}(0)}{r} \frac{\partial^{2} V_{3}}{\partial \rho \partial r}-\left(\frac{\rho^{\prime}(0)}{\rho(0)} \frac{\partial^{2} V_{3}}{\partial \rho^{2}}-\frac{\rho^{\prime}(0)}{\rho^{2}(0)} \frac{\partial V_{3}}{\partial \rho}\right)\right] \varepsilon
\end{aligned}
$$

Then substituting the latter expression into equation (2.7), we obtain the two differential equations

$$
\begin{aligned}
r^{\prime}= & -\frac{1}{r}\left(\frac{\partial V_{3}}{\partial \theta}-\frac{\partial V_{3}}{\partial \alpha}\right) \varepsilon+\frac{1}{r}\left[\frac{1}{r} \frac{\partial V_{3}}{\partial r}\left(\frac{\partial V_{3}}{\partial \theta}-\frac{\partial V_{3}}{\partial \alpha}\right)-\rho^{\prime}(0)\left(\frac{\partial^{2} V_{3}}{\partial \rho \partial \theta}-\frac{\partial^{2} V_{3}}{\partial \rho \partial \alpha}\right)-\right. \\
& \left.\left(\frac{\partial V_{4}}{\partial \theta}-\frac{\partial V_{4}}{\partial \alpha}\right)\right] \varepsilon^{2}+O\left(\varepsilon^{3}\right) \\
\alpha^{\prime}= & -\frac{1}{r}\left(\frac{\partial V_{3}}{\partial r}-\frac{r}{\rho(0)} \frac{\partial V_{3}}{\partial \rho}\right) \varepsilon+\left[-\frac{\rho^{\prime}(0)}{r} \frac{\partial^{2} V_{3}}{\partial \rho \partial r}+\left(\frac{\rho^{\prime}(0)}{\rho(0)} \frac{\partial^{2} V_{3}}{\partial \rho^{2}}-\frac{\rho^{\prime}(0)}{\rho^{2}(0)} \frac{\partial V_{3}}{\partial \rho}\right)+\right. \\
& \left.\frac{1}{r} \frac{\partial V_{3}}{\partial r}\left(\frac{1}{r} \frac{\partial V_{3}}{\partial r}-\frac{1}{\rho} \frac{\partial V_{3}}{\partial \rho}\right)-\left(\frac{1}{r} \frac{\partial V_{4}}{\partial r}-\frac{1}{\rho} \frac{\partial V_{4}}{\partial \rho}\right)\right] \varepsilon^{2}+O\left(\varepsilon^{3}\right)
\end{aligned}
$$

where the partial derivatives of $V_{3}$ and $V_{4}$ are evaluated at the point $\rho=\rho(0)$ (as in (2.9) and $(r \cos \theta, \rho(0) \cos (\theta+\alpha))$.

The system (2.12) has the general form

$$
\begin{aligned}
r^{\prime} & =F_{11} \varepsilon+F_{21} \varepsilon^{2}+O\left(\varepsilon^{3}\right), \\
\alpha^{\prime} & =F_{12} \varepsilon+F_{22} \varepsilon^{2}+O\left(\varepsilon^{3}\right),
\end{aligned}
$$


where

$$
\begin{aligned}
F_{11}= & -\frac{1}{r}\left(\frac{\partial V_{3}}{\partial \theta}-\frac{\partial V_{3}}{\partial \alpha}\right), \\
F_{12}= & -\frac{1}{r}\left(\frac{\partial V_{3}}{\partial r}-\frac{r}{\rho(0)} \frac{\partial V_{3}}{\partial \rho}\right), \\
F_{21}= & \frac{1}{r}\left[\frac{1}{r} \frac{\partial V_{3}}{\partial r}\left(\frac{\partial V_{3}}{\partial \theta}-\frac{\partial V_{3}}{\partial \alpha}\right)-\rho^{\prime}(0)\left(\frac{\partial^{2} V_{3}}{\partial \rho \partial \theta}-\frac{\partial^{2} V_{3}}{\partial \rho \partial \alpha}\right)-\right. \\
& \left.\left(\frac{\partial V_{4}}{\partial \theta}-\frac{\partial V_{4}}{\partial \alpha}\right)\right], \\
F_{22}= & {\left[-\frac{\rho^{\prime}(0)}{r} \frac{\partial^{2} V_{3}}{\partial \rho \partial r}+\left(\frac{\rho^{\prime}(0)}{\rho(0)} \frac{\partial^{2} V_{3}}{\partial \rho^{2}}-\frac{\rho^{\prime}(0)}{\rho^{2}(0)} \frac{\partial V_{3}}{\partial \rho}\right)+\right.} \\
& \left.\frac{1}{r} \frac{\partial V_{3}}{\partial r}\left(\frac{1}{r} \frac{\partial V_{3}}{\partial r}-\frac{1}{\rho} \frac{\partial V_{3}}{\partial \rho}\right)-\left(\frac{1}{r} \frac{\partial V_{4}}{\partial r}-\frac{1}{\rho} \frac{\partial V_{4}}{\partial \rho}\right)\right] .
\end{aligned}
$$

Also, we denote

$$
f_{1}(r, \alpha):=\left(f_{11}, f_{12}\right)=\frac{1}{2 \pi} \int_{0}^{2 \pi}\left(F_{11}, F_{12}\right) d \theta
$$

and

$$
\begin{aligned}
f_{2}(r, \alpha) & :=\left(f_{21}, f_{22}\right) \\
& =\frac{1}{2 \pi} \int_{0}^{2 \pi}\left[D_{r \alpha} F_{1}(\theta, r, \alpha) \cdot \int_{0}^{\theta} F_{1}(t, r, \alpha) d t+F_{2}(\theta, r, \alpha)\right] d \theta,
\end{aligned}
$$

where $F_{1}=\left(F_{11}, F_{12}\right), F_{2}=\left(F_{21}, F_{22}\right)$ and $D_{r \alpha} F_{1}(\theta, r, \alpha)$ is the Jacobian matrix given by

$$
D_{r \alpha} F_{1}(\theta, r, \alpha)=\left(\begin{array}{ll}
\frac{\partial F_{11}}{\partial r} & \frac{\partial F_{11}}{\partial \alpha} \\
\frac{\partial F_{12}}{\partial r} & \frac{\partial F_{12}}{\partial \alpha}
\end{array}\right) .
$$

The following lemma is related to the expressions (2.13), (2.15), (2.16) and will be very useful for future computations.

Lemma 2.1. $f_{1}(r, \alpha)=(0,0)$

Proof. Note that

$$
\begin{aligned}
& F_{11}=\left[B\left(2 h-r^{2}\right) \cos ^{2}(\alpha+\theta)+A r^{2} \cos ^{2} \theta\right] \sin \theta, \\
& F_{12}=-\frac{\left[B\left(2 h-3 r^{2}\right) \cos ^{2}(\alpha+\theta)+A r^{2} \cos ^{2} \theta\right] \cos \theta}{r} .
\end{aligned}
$$

As each of the integrals in (2.15) involves the presence of combinations of the functions $\cos ^{3}(\theta)$, $\cos ^{2}(\theta) \sin (\theta), \cos (\theta) \sin ^{2}(\theta), \sin ^{3}(\theta)$, we arrive to the equation $f_{1}(r, \alpha) \equiv(0,0)$. 


\section{Statement of the problem and equations of motion for $H^{-}$}

The equations of motion associated to the system (1.2) are

$$
\begin{aligned}
\dot{x} & =-p_{x} \\
\dot{y} & =p_{y}, \\
\dot{p}_{x} & =x-\frac{\partial V_{3}}{\partial x}-\frac{\partial V_{4}}{\partial x}, \\
\dot{p}_{y} & =-y-\frac{\partial V_{3}}{\partial y}-\frac{\partial V_{4}}{\partial y} .
\end{aligned}
$$

Let $x=\sqrt{\varepsilon} X, y=\sqrt{\varepsilon} Y, p_{x}=\sqrt{\varepsilon} p_{X}$ and $p_{y}=\sqrt{\varepsilon} p_{Y}$ be the change of variable, which is $\varepsilon^{-2}$ symplectic, therefore the system (3.1) becomes

$$
\begin{aligned}
\dot{X} & =-p_{X}, \\
\dot{Y} & =p_{Y}, \\
\dot{p}_{X} & =X-\varepsilon \frac{\partial V_{3}}{\partial X}-\varepsilon^{2} \frac{\partial V_{4}}{\partial X}, \\
\dot{p}_{Y} & =-Y-\varepsilon \frac{\partial V_{3}}{\partial Y}-\varepsilon^{2} \frac{\partial V_{4}}{\partial Y} .
\end{aligned}
$$

The Hamiltonian function associated to (3.1) and (3.2) is

$$
K^{-}=\frac{1}{2}\left(-p_{X}^{2}+p_{Y}^{2}\right)+\frac{1}{2}\left(-X^{2}+Y^{2}\right)+\varepsilon V_{3}+\varepsilon^{2} V_{4}
$$

where $K^{-}=\varepsilon^{-2} H^{-}=h$.

By the standard theory of Hamiltonian dynamical systems, for all $\varepsilon$ different from zero, the original system (3.1) and the new system (3.2) have essentially the same phase portrait, and additionally the system (3.2), for $\varepsilon$ sufficiently small, is close to an integrable system.

Now, analogously to the previous case we introduce the convenient change of coordinates $(r, \rho, \theta, \alpha)$ by the relations

$$
X=r \cos \theta, \quad Y=\rho \cos (-\theta+\alpha), \quad p_{X}=r \sin \theta, \quad p_{Y}=\rho \sin (-\theta+\alpha) .
$$

Recall that this is a well defined change of variables when $r>0$ and $\rho>0$. We observe that for a fixed value $h$ of $K$, in polar coordinates,

$$
h=\frac{1}{2}\left(-r^{2}+\rho^{2}\right)+\varepsilon V_{3}(r \cos \theta, \rho \cos (-\theta+\alpha))+\varepsilon^{2} V_{4}(r \cos \theta, \rho \cos (-\theta+\alpha)) .
$$


Differentiating directly, we arrive to the fact that the equations of motion assume the form

$$
\begin{aligned}
& \dot{r}=-\varepsilon \sin \theta \frac{\partial V_{3}}{\partial X}-\varepsilon^{2} \sin \theta \frac{\partial V_{4}}{\partial X}, \\
& \dot{\theta}=1-\varepsilon \frac{\cos \theta}{r} \frac{\partial V_{3}}{\partial X}-\varepsilon^{2} \frac{\cos \theta}{r} \frac{\partial V_{4}}{\partial X}, \\
& \dot{\rho}=\varepsilon \sin (-\theta+\alpha) \frac{\partial V_{3}}{\partial Y}+\varepsilon^{2} \sin (-\theta+\alpha) \frac{\partial V_{4}}{\partial Y}, \\
& \dot{\alpha}=\varepsilon\left[-\frac{\cos \theta}{r} \frac{\partial V_{3}}{\partial X}-\frac{\cos (-\theta+\alpha)}{\rho} \frac{\partial V_{3}}{\partial Y}\right]+\varepsilon^{2}\left[-\frac{\cos \theta}{r} \frac{\partial V_{4}}{\partial X}-\frac{\cos (-\theta+\alpha)}{\rho} \frac{\partial V_{4}}{\partial Y}\right] .
\end{aligned}
$$

Moreover, note that

$$
\begin{aligned}
& \frac{\partial V_{k}}{\partial r}=\cos \theta \frac{\partial V_{k}}{\partial X}, \\
& \frac{\partial V_{k}}{\partial \theta}=-r \sin \theta \frac{\partial V_{k}}{\partial X}+\rho \sin (-\theta+\alpha) \frac{\partial V_{k}}{\partial Y}, \\
& \frac{\partial V_{k}}{\partial \rho}=\cos (-\theta+\alpha) \frac{\partial V_{k}}{\partial Y}, \\
& \frac{\partial V_{k}}{\partial \alpha}=-\rho \sin (-\theta+\alpha) \frac{\partial V_{k}}{\partial Y} .
\end{aligned}
$$

for $k=3,4$. Therefore (2.4) can be written as

$$
\begin{aligned}
& \dot{r}=\varepsilon \frac{1}{r}\left(\frac{\partial V_{3}}{\partial \theta}+\frac{\partial V_{3}}{\partial \alpha}\right)+\varepsilon^{2} \frac{1}{r}\left(\frac{\partial V_{4}}{\partial \theta}+\frac{\partial V_{4}}{\partial \alpha}\right), \\
& \dot{\theta}=1-\varepsilon \frac{1}{r} \frac{\partial V_{3}}{\partial r}-\varepsilon^{2} \frac{1}{r} \frac{\partial V_{4}}{\partial r}, \\
& \dot{\rho}=-\varepsilon \frac{1}{\rho} \frac{\partial V_{3}}{\partial \alpha}-\varepsilon^{2} \frac{1}{\rho} \frac{\partial V_{4}}{\partial \alpha}, \\
& \dot{\alpha}=\varepsilon\left(-\frac{1}{r} \frac{\partial V_{3}}{\partial r}-\frac{1}{\rho} \frac{\partial V_{3}}{\partial \rho}\right)+\varepsilon^{2}\left(-\frac{1}{r} \frac{\partial V_{4}}{\partial r}-\frac{1}{\rho} \frac{\partial V_{4}}{\partial \rho}\right),
\end{aligned}
$$

where the partial derivatives of $V_{3}$ and $V_{4}$ are evaluated at the point $(r \cos \theta, \rho \cos (-\theta+\alpha))$. In order to put our system as a periodic system of ordinary differential equations, we introduce the $\theta$ variable as independent (new time), and we use the notation prime to denote the derivative with respect to $\theta$. It is observed that the angular variable $\alpha$ cannot be used as the independent variable, since the new differential system does not have the appropriate form in order to apply the averaging method. Now, note that

$$
\frac{d r}{d \theta}=\frac{\varepsilon\left(\frac{\partial V_{3}}{\partial \theta}+\frac{\partial V_{3}}{\partial \alpha}\right)+\varepsilon^{2}\left(\frac{\partial V_{4}}{\partial \theta}+\frac{\partial V_{4}}{\partial \alpha}\right)}{r-\varepsilon \frac{\partial V_{3}}{\partial r}-\varepsilon^{2} \frac{\partial V_{4}}{\partial r}} .
$$

Remember that the function

$$
f(\varepsilon)=\frac{1}{1-a_{1} \varepsilon-a_{2} \varepsilon^{2}}=1+a_{1} \varepsilon+\left(a_{1}^{2}+a_{2}\right) \varepsilon^{2}+O\left(\varepsilon^{3}\right),
$$


by developing a Taylor series in powers of $\varepsilon$ around $\varepsilon=0$. Replacing $a_{1}=\frac{1}{r} \frac{\partial V_{3}}{\partial r}$ and $a_{2}=\frac{1}{r} \frac{\partial V_{4}}{\partial r}$ we get

$$
\frac{1}{1-\frac{1}{r} \frac{\partial V_{3}}{\partial r} \varepsilon-\frac{1}{r} \frac{\partial V_{4}}{\partial r} \varepsilon^{2}}=1+\frac{1}{r} \frac{\partial V_{3}}{\partial r} \varepsilon+\left[\left(\frac{1}{r} \frac{\partial V_{3}}{\partial r}\right)^{2}+\frac{1}{r} \frac{\partial V_{4}}{\partial r}\right] \varepsilon^{2}+O\left(\varepsilon^{3}\right) .
$$

Therefore,

$$
r^{\prime}=\frac{1}{r}\left(\frac{\partial V_{3}}{\partial \theta}+\frac{\partial V_{3}}{\partial \alpha}\right) \varepsilon+\frac{1}{r}\left[\frac{1}{r} \frac{\partial V_{3}}{\partial r}\left(\frac{\partial V_{3}}{\partial \theta}+\frac{\partial V_{3}}{\partial \alpha}\right)+\left(\frac{\partial V_{4}}{\partial \theta}+\frac{\partial V_{4}}{\partial \alpha}\right)\right] \varepsilon^{2}+O\left(\varepsilon^{3}\right) .
$$

Moreover,

$$
\frac{d \rho}{d \theta}=-\frac{\varepsilon \frac{1}{\rho} \frac{\partial V_{3}}{\partial \alpha}+\varepsilon^{2} \frac{1}{\rho} \frac{\partial V_{4}}{\partial \alpha}}{1-\varepsilon \frac{1}{r} \frac{\partial V_{3}}{\partial r}-\varepsilon^{2} \frac{1}{r} \frac{\partial V_{4}}{\partial r}}
$$

thus

$$
\rho^{\prime}=-\frac{1}{\rho} \frac{\partial V_{3}}{\partial \alpha} \varepsilon-\left[\frac{1}{r \rho} \frac{\partial V_{3}}{\partial r} \frac{\partial V_{3}}{\partial \alpha}+\frac{1}{\rho} \frac{\partial V_{4}}{\partial \alpha}\right] \varepsilon^{2}+O\left(\varepsilon^{3}\right)
$$

Also

$$
\frac{d \alpha}{d \theta}=\frac{\varepsilon\left(-\frac{1}{r} \frac{\partial V_{3}}{\partial r}-\frac{1}{\rho} \frac{\partial V_{3}}{\partial \rho}\right)+\varepsilon^{2}\left(-\frac{1}{r} \frac{\partial V_{4}}{\partial r}-\frac{1}{\rho} \frac{\partial V_{4}}{\partial \rho}\right)}{1-\varepsilon \frac{1}{r} \frac{\partial V_{3}}{\partial r}-\varepsilon^{2} \frac{1}{r} \frac{\partial V_{4}}{\partial r}}
$$

then

$$
\alpha^{\prime}=\left(-\frac{1}{r} \frac{\partial V_{3}}{\partial r}-\frac{1}{\rho} \frac{\partial V_{3}}{\partial \rho}\right) \varepsilon+\left[\frac{1}{r} \frac{\partial V_{3}}{\partial r}\left(-\frac{1}{r} \frac{\partial V_{3}}{\partial r}-\frac{1}{\rho} \frac{\partial V_{3}}{\partial \rho}\right)+\left(-\frac{1}{r} \frac{\partial V_{4}}{\partial r}-\frac{1}{\rho} \frac{\partial V_{4}}{\partial \rho}\right)\right] \varepsilon^{2}+O\left(\varepsilon^{3}\right) .
$$

If we write the previous system as a Taylor series in powers of $\varepsilon$, we have that

$$
\begin{aligned}
r^{\prime}= & \frac{1}{r}\left(\frac{\partial V_{3}}{\partial \theta}+\frac{\partial V_{3}}{\partial \alpha}\right) \varepsilon+\frac{1}{r}\left[\frac{1}{r} \frac{\partial V_{3}}{\partial r}\left(\frac{\partial V_{3}}{\partial \theta}+\frac{\partial V_{3}}{\partial \alpha}\right)+\left(\frac{\partial V_{4}}{\partial \theta}+\frac{\partial V_{4}}{\partial \alpha}\right)\right] \varepsilon^{2}+O\left(\varepsilon^{3}\right), \\
\rho^{\prime}= & -\frac{1}{\rho} \frac{\partial V_{3}}{\partial \alpha} \varepsilon-\left[\frac{1}{r \rho} \frac{\partial V_{3}}{\partial r} \frac{\partial V_{3}}{\partial \alpha}+\frac{1}{\rho} \frac{\partial V_{4}}{\partial \alpha}\right] \varepsilon^{2}+O\left(\varepsilon^{3}\right), \\
\alpha^{\prime}= & \left(-\frac{1}{r} \frac{\partial V_{3}}{\partial r}-\frac{1}{\rho} \frac{\partial V_{3}}{\partial \rho}\right) \varepsilon+\left[\frac{1}{r} \frac{\partial V_{3}}{\partial r}\left(-\frac{1}{r} \frac{\partial V_{3}}{\partial r}-\frac{1}{\rho} \frac{\partial V_{3}}{\partial \rho}\right)+\left(-\frac{1}{r} \frac{\partial V_{4}}{\partial r}-\frac{1}{\rho} \frac{\partial V_{4}}{\partial \rho}\right)\right] \varepsilon^{2}+ \\
& O\left(\varepsilon^{3}\right) .
\end{aligned}
$$

In order to apply the averaging theory we will fix the value of the first integral of $K=h>0$. We write the Taylor expansion of the function $\rho$ of second order in $\varepsilon$ and rewrite the system (3.7) in the 
variables $r$ and $\alpha$. The function $\rho$ in its Taylor form is

$$
\rho(\varepsilon)=\rho(0)+\rho^{\prime}(0) \varepsilon+O\left(\varepsilon^{2}\right) .
$$

Regarding (3.4), we obtain for $\varepsilon=0$,

$$
\rho(0)=\sqrt{2 h+r^{2}} .
$$

Derivating (3.4) with respect to $\varepsilon$, we have

$$
\begin{aligned}
0= & \rho \rho^{\prime}+V_{3}(r \cos \theta, \rho \cos (-\theta+\alpha))+\varepsilon \frac{\partial V_{3}}{\partial Y} \rho^{\prime} \cos (-\theta+\alpha)+ \\
& 2 \varepsilon V_{4}(r \cos \theta, \rho \cos (-\theta+\alpha))+\varepsilon^{2} \frac{\partial V_{4}}{\partial Y} \rho^{\prime} \cos (-\theta+\alpha)
\end{aligned}
$$

and evaluating at $\varepsilon=0$, we obtain

$$
\rho^{\prime}(0)=-\frac{1}{\sqrt{2 h+r^{2}}} V_{3}\left(r \cos \theta, \sqrt{2 h+r^{2}} \cos (-\theta+\alpha)\right) .
$$

Thus, from (3.8), (3.9) and (3.10) the development of the function $\rho$ in a Taylor series has the form

$$
\rho(\varepsilon)=\sqrt{2 h+r^{2}}-\frac{1}{\sqrt{2 h+r^{2}}} V_{3}\left(r \cos \theta, \sqrt{2 h+r^{2}} \cos (-\theta+\alpha)\right) \varepsilon+O\left(\varepsilon^{2}\right) .
$$

Since $V_{3}$ depends on the variables $(r, \rho, \theta, \alpha)$, the derivatives of $V_{3}$ respect to their variables in the Taylor series are:

- $\frac{\partial V_{3}}{\partial \theta}=\frac{\partial V_{3}}{\partial \theta}+\rho^{\prime}(0) \frac{\partial^{2} V_{3}}{\partial \rho \partial \theta} \varepsilon+O\left(\varepsilon^{2}\right)$,

- $\frac{\partial V_{3}}{\partial \alpha}=\frac{\partial V_{3}}{\partial \alpha}+\rho^{\prime}(0) \frac{\partial^{2} V_{3}}{\partial \rho \partial \alpha} \varepsilon+O\left(\varepsilon^{2}\right)$

- $\frac{\partial V_{3}}{\partial r}=\frac{\partial V_{3}}{\partial r}+\rho^{\prime}(0) \frac{\partial^{2} V_{3}}{\partial \rho \partial r} \varepsilon+O\left(\varepsilon^{2}\right)$,

- $\frac{\partial V_{3}}{\partial \rho}=\frac{\partial V_{3}}{\partial \rho}+\rho^{\prime}(0) \frac{\partial^{2} V_{3}}{\partial \rho^{2}} \varepsilon+O\left(\varepsilon^{2}\right)$.

Moreover,

- $\frac{1}{\rho}=\frac{1}{\rho(0)}-\frac{\rho^{\prime}(0)}{\rho^{2}(0)} \varepsilon+O\left(\varepsilon^{2}\right)$,

- $\frac{1}{\rho} \frac{\partial V_{3}}{\partial \rho}=\frac{1}{\rho(0)} \frac{\partial V_{3}}{\partial \rho}+\left[\frac{\rho^{\prime}(0)}{\rho(0)} \frac{\partial^{2} V_{3}}{\partial \rho^{2}}-\frac{\rho^{\prime}(0)}{\rho^{2}(0)} \frac{\partial V_{3}}{\partial \rho}\right] \varepsilon+O\left(\varepsilon^{2}\right)$. 
Recall that all the previous functions are evaluated at the point $(r \cos \theta, \rho(0) \cos (-\theta+\alpha))$. Therefore,

$$
\begin{aligned}
\frac{1}{r}\left(\frac{\partial V_{3}}{\partial \theta}+\frac{\partial V_{3}}{\partial \alpha}\right) & =\frac{1}{r}\left(\frac{\partial V_{3}}{\partial \theta}+\frac{\partial V_{3}}{\partial \alpha}\right)+\frac{\rho^{\prime}(0)}{r}\left(\frac{\partial^{2} V_{3}}{\partial \rho \partial \theta}+\frac{\partial^{2} V_{3}}{\partial \rho \partial \alpha}\right) \varepsilon \\
\left(-\frac{1}{r} \frac{\partial V_{3}}{\partial r}-\frac{1}{\rho} \frac{\partial V_{3}}{\partial \rho}\right) & =\left(-\frac{1}{r} \frac{\partial V_{3}}{\partial r}-\frac{1}{\rho(0)} \frac{\partial V_{3}}{\partial \rho}\right)-\left[\frac{\rho^{\prime}(0)}{r} \frac{\partial^{2} V_{3}}{\partial \rho \partial r}+\left(\frac{\rho^{\prime}(0)}{\rho(0)} \frac{\partial^{2} V_{3}}{\partial \rho^{2}}-\frac{\rho^{\prime}(0)}{\rho^{2}(0)} \frac{\partial V_{3}}{\partial \rho}\right)\right] \varepsilon .
\end{aligned}
$$

Then substituting the latter expression into equation (3.7), we obtain the two differential equations

$$
\begin{aligned}
r^{\prime}= & \frac{1}{r}\left(\frac{\partial V_{3}}{\partial \theta}+\frac{\partial V_{3}}{\partial \alpha}\right) \varepsilon+\frac{1}{r}\left[\frac{1}{r} \frac{\partial V_{3}}{\partial r}\left(\frac{\partial V_{3}}{\partial \theta}+\frac{\partial V_{3}}{\partial \alpha}\right)+\rho^{\prime}(0)\left(\frac{\partial^{2} V_{3}}{\partial \rho \partial \theta}+\frac{\partial^{2} V_{3}}{\partial \rho \partial \alpha}\right)+\right. \\
& \left.\left(\frac{\partial V_{4}}{\partial \theta}+\frac{\partial V_{4}}{\partial \alpha}\right)\right] \varepsilon^{2}+O\left(\varepsilon^{3}\right), \\
\alpha^{\prime}= & \frac{1}{r}\left(-\frac{\partial V_{3}}{\partial r}-\frac{r}{\rho(0)} \frac{\partial V_{3}}{\partial \rho}\right) \varepsilon+\left[-\frac{\rho^{\prime}(0)}{r} \frac{\partial^{2} V_{3}}{\partial \rho \partial r}-\left(\frac{\rho^{\prime}(0)}{\rho(0)} \frac{\partial^{2} V_{3}}{\partial \rho^{2}}-\frac{\rho^{\prime}(0)}{\rho^{2}(0)} \frac{\partial V_{3}}{\partial \rho}\right)+\right. \\
& \left.\frac{1}{r} \frac{\partial V_{3}}{\partial r}\left(-\frac{1}{r} \frac{\partial V_{3}}{\partial r}-\frac{1}{\rho(0)} \frac{\partial V_{3}}{\partial \rho}\right)+\left(-\frac{1}{r} \frac{\partial V_{4}}{\partial r}-\frac{1}{\rho(0)} \frac{\partial V_{4}}{\partial \rho}\right)\right] \varepsilon^{2}+O\left(\varepsilon^{3}\right),
\end{aligned}
$$

where the last system is evaluated at the point $\rho=\rho(0)$ as in (3.9) and $(r \cos \theta, \rho(0) \cos (-\theta+\alpha))$.

The system (3.12) has the general form

$$
\begin{aligned}
r^{\prime} & =F_{11} \varepsilon+F_{21} \varepsilon^{2}+O\left(\varepsilon^{3}\right), \\
\alpha^{\prime} & =F_{12} \varepsilon+F_{22} \varepsilon^{2}+O\left(\varepsilon^{3}\right),
\end{aligned}
$$

where

$$
\begin{aligned}
F_{11}= & \frac{1}{r}\left(\frac{\partial V_{3}}{\partial \theta}+\frac{\partial V_{3}}{\partial \alpha}\right), \\
F_{12}= & -\frac{1}{r}\left(\frac{\partial V_{3}}{\partial r}+\frac{r}{\rho(0)} \frac{\partial V_{3}}{\partial \rho}\right), \\
F_{21}= & \frac{1}{r}\left[\frac{1}{r} \frac{\partial V_{3}}{\partial r}\left(\frac{\partial V_{3}}{\partial \theta}+\frac{\partial V_{3}}{\partial \alpha}\right)+\rho^{\prime}(0)\left(\frac{\partial^{2} V_{3}}{\partial \rho \partial \theta}+\frac{\partial^{2} V_{3}}{\partial \rho \partial \alpha}\right)+\right. \\
& \left.\left(\frac{\partial V_{4}}{\partial \theta}+\frac{\partial V_{4}}{\partial \alpha}\right)\right], \\
F_{22}= & {\left[-\frac{\rho^{\prime}(0)}{r} \frac{\partial^{2} V_{3}}{\partial \rho \partial r}-\left(\frac{\rho^{\prime}(0)}{\rho(0)} \frac{\partial^{2} V_{3}}{\partial \rho^{2}}-\frac{\rho^{\prime}(0)}{\rho^{2}(0)} \frac{\partial V_{3}}{\partial \rho}\right)+\right.} \\
& \left.\frac{1}{r} \frac{\partial V_{3}}{\partial r}\left(-\frac{1}{r} \frac{\partial V_{3}}{\partial r}-\frac{1}{\rho} \frac{\partial V_{3}}{\partial \rho}\right)+\left(-\frac{1}{r} \frac{\partial V_{4}}{\partial r}-\frac{1}{\rho} \frac{\partial V_{4}}{\partial \rho}\right)\right] .
\end{aligned}
$$

Also, we denote

$$
f_{1}(r, \alpha):=\left(f_{11}, f_{12}\right)=\frac{1}{2 \pi} \int_{0}^{2 \pi}\left(F_{11}, F_{12}\right) d \theta
$$


and

$$
\begin{aligned}
f_{2}(r, \alpha) & :=\left(f_{21}, f_{22}\right) \\
& =\frac{1}{2 \pi} \int_{0}^{2 \pi}\left[D_{r \alpha} F_{1}(\theta, r, \alpha) \cdot \int_{0}^{\theta} F_{1}(t, r, \alpha) d t+F_{2}(\theta, r, \alpha)\right] d \theta,
\end{aligned}
$$

where $F_{1}=\left(F_{11}, F_{12}\right), F_{2}=\left(F_{21}, F_{22}\right)$ and $D_{r \alpha} F_{1}(\theta, r, \alpha)$ is the jacobian matrix given by

$$
D_{r \alpha} F_{1}(\theta, r, \alpha)=\left(\begin{array}{ll}
\frac{\partial F_{11}}{\partial r} & \frac{\partial F_{11}}{\partial \alpha} \\
\frac{\partial F_{12}}{\partial r} & \frac{\partial F_{12}}{\partial \alpha}
\end{array}\right) .
$$

As the expression given in $F_{11}$ and $F_{22}$ involves the presence of combinations of the functions $\cos ^{3} \theta$, $\cos ^{2} \theta \sin \theta, \cos \theta \sin ^{2} \theta, \sin ^{3} \theta$, we arrive to the equation $f_{1}(r, \alpha)=(0,0)$. This result is the same as in Lemma 2.1 .

\section{Proof of Theorem 1.1 and Theorem 1.2, the case $H^{+}$}

Proof. We use the same notation as in [6] to apply the averaging method. Let $H$ be as in (1.1) such that $h$ be any small positive number. Moreover, the expressions of $f_{21}$ and $f_{22}$ given by (2.16) are of the form:

$$
\begin{aligned}
f_{21}= & \frac{1}{24} r\left(r^{2}-2 h\right)\left[2 A B+3\left(m-4 B^{2}\right)\right] \sin (2 \alpha) \\
f_{22}= & \frac{1}{24}\left[10 A^{2} r^{2}+24 A B\left(h-r^{2}\right)-2 B^{2}\left(2 h+3 r^{2}\right)-3\left(h(4 m-6 \lambda)+r^{2}(-4 m+3(\lambda+\Lambda))\right)-\right. \\
& \left.2(2(A-6 B) B+3 m)\left(h-r^{2}\right) \cos (2 \alpha)\right] .
\end{aligned}
$$

Let $M_{j}$, for $j=1, \ldots, 7$ be the expressions given in Section 1 . In order to apply the averaging method, first we solve the system $f_{21}=0$ and $f_{22}=0$ for the variables $r$ and $\alpha$. Therefore, we obtain the solutions $\left(r_{j}, \alpha_{j}\right)$, for $j=1,2,3$, given by

$$
\begin{aligned}
& \left(r_{1}, \alpha_{1}\right)=\left(\sqrt{-2 h \frac{M_{1}}{M_{2}}}, 0\right), \\
& \left(r_{2}, \alpha_{2}\right)=\left(\sqrt{-2 h \frac{M_{5}}{M_{6}}},-\frac{\pi}{2}\right), \\
& \left(r_{2}, \alpha_{2}\right)=\left(r_{2}, \frac{\pi}{2}\right) .
\end{aligned}
$$

Remember that $\rho_{j}=\sqrt{2 h-r_{j}^{2}}$, for $j=1,2,3$. Therefore, this expressions are

$$
\begin{aligned}
& \rho_{1}=\sqrt{2 h \frac{M_{3}}{M_{2}}}, \\
& \rho_{2}=\sqrt{2 h \frac{M_{7}}{M_{6}}} . \\
& \rho_{3}=\rho_{2}
\end{aligned}
$$


The next step is to find the region of the parameters where the determinant $J_{j}$ of the Jacobian matrix, $D_{r, \alpha}\left(f_{1}, f_{2}\right)$ evaluated in the zeros $\left(r_{j}, \alpha_{j}\right)$ is non null. We find that

$$
\begin{aligned}
& J_{1}=\frac{h r_{1}^{2}}{72} M_{3} M_{4}, \\
& J_{2}=-\frac{h r_{2}^{2}}{72} M_{4} M_{7}, \\
& J_{3}=J_{2} .
\end{aligned}
$$

Now, we finish the proof of Theorem 1.1. Indeed, if $(A, B, m, \lambda, \Lambda) \in \Lambda_{h}^{1} \backslash \Lambda_{h}^{2}$, then $r_{1}>0, \rho_{1}>0$ and $J_{1} \neq 0$ and therefore we obtain at least one-family of period orbits. If $(A, B, m, \lambda, \Lambda) \in \Lambda_{h}^{2} \backslash \Lambda_{h}^{1}$, then $r_{2}>0, \rho_{2}>0$ and $J_{2} \neq 0$, so we get at least two-families of periodic orbits. Finally, if $(A, B, m, \lambda, \Lambda) \in \Lambda_{h}^{1} \cap \Lambda_{h}^{2}$, then $r_{1}>0, r_{2}>0, \rho_{1}>0, \rho_{2}>0, J_{1} \neq 0$ and $J_{2} \neq 0$, so we get at least three-families of periodic orbits. This completes the proof of Theorem 1.1.

For the proof of Theorem 1.2. Observe that if we denote by $\lambda_{1}^{1}$ and $\lambda_{2}^{1}$ the eigenvalued of the Jacobian matrix $D_{r, \alpha}\left(f_{1}, f_{2}\right)$ evaluate in $\left(r_{1}, \alpha_{1}\right)$, we have that

$$
\lambda_{1}^{1}=-\frac{1}{6 \sqrt{2}} r_{1} \sqrt{h} \sqrt{-M_{3} M_{4}}, \lambda_{2}^{1}=-\lambda_{1}^{1}
$$

Moreover, as $J_{1}=\lambda_{1}^{1} \lambda_{2}^{1}$, we obtain that $J_{1}=-\left(\lambda_{1}^{1}\right)^{2}$. Therefore, for $(A, B, m, \lambda, \Lambda) \in \Lambda_{h}^{1} \backslash \Lambda_{h}^{2}$ such that $M_{3} M_{4}>0$ we obtain at least one-family linearly stable and linearly unstable if $M_{3} M_{4}<0$. This proves the first part of Theorem 1.2. For the second part of Theorem 1.2, we denote by $\lambda_{1}^{2}$ and $\lambda_{2}^{2}$ the eigenvalues of the Jacobian matrix $D_{r, \alpha}\left(f_{1}, f_{2}\right)$ evaluated in $\left(r_{2}, \alpha_{2}\right)$, we have that

$$
\lambda_{1}^{2}=-\frac{1}{6 \sqrt{2}} r_{2} \sqrt{h} \sqrt{M_{4} M_{7}}, \lambda_{2}^{2}=-\lambda_{1}^{2} .
$$

Moreover, as $J_{2}=\lambda_{1}^{2} \lambda_{2}^{2}$, we obtain that $J_{2}=-\left(\lambda_{1}^{2}\right)^{2}$. Therefore, for $(A, B, m, \lambda, \Lambda) \in \Lambda_{h}^{2} \backslash \Lambda_{h}^{1}$ such that $M_{4} M_{7}<0$ we obtain at least two-families linearly stable and linearly unstable if $M_{4} M_{7}>0$. Finally, for the proof of the third part of Theorem 1.2, for $(A, B, m, \lambda, \Lambda) \in \Lambda_{h}^{1} \cap \Lambda_{h}^{2}$ such that $M_{3} M_{4}>$ 0 and $M_{4} M_{7}<0$, we obtain three-families linearly stable and three-families linearly unstable in the other conditions. Therefore, the proof is completed.

\section{Proof of Theorem 1.3 and Theorem 1.4, the case $H^{-}$}

Proof. We use the same notation as in [6] to apply the averaging method. Let $H$ be as in (1.2) such that $h$ be any small number. Moreover, the functions $f_{21}$ and $f_{22}$ given by (3.16) are:

$$
\begin{aligned}
f_{21}= & \frac{1}{24} r\left(2 h+r^{2}\right)\left(2 A B+12 B^{2}-3 m\right) \sin (2 \alpha), \\
f_{22}= & \frac{1}{24}\left[-10 A^{2} r^{2}-24 A B\left(h+r^{2}\right)+B^{2}\left(-4 h+6 r^{2}\right)-\right. \\
& 3\left(h(4 m+6 \lambda)+r^{2}(4 m+3(\lambda+\Lambda))\right)+ \\
& \left.2(2 B(A+6 B)-3 m)\left(h+r^{2}\right) \cos (2 \alpha)\right] .
\end{aligned}
$$

Let $N_{j}$ be for $j=1, \ldots, 7$ the expressions given in Section 1 . To apply the averaging method, first we solve the system $f_{21}=0$ and $f_{22}=0$ for the variables $r$ and $\alpha$. Therefore, we obtain the solutions 
$\left(r_{j}, \alpha_{j}\right)$, for $j=1,2,3$, given by

$$
\begin{aligned}
& \left(r_{1}, \alpha_{1}\right)=\left(\sqrt{-2 h \frac{N_{1}}{N_{2}}}, 0\right), \\
& \left(r_{2}, \alpha_{2}\right)=\left(\sqrt{-2 h \frac{N_{5}}{N_{6}}},-\frac{\pi}{2}\right), \\
& \left(r_{3}, \alpha_{3}\right)=\left(r_{2}, \frac{\pi}{2}\right) .
\end{aligned}
$$

Remember that in the case $H^{-}, \rho_{j}=\sqrt{2 h+r_{j}^{2}}$, for $j=1,2,3$. Therefore, these expressions are

$$
\begin{aligned}
& \rho_{1}=\sqrt{2 h \frac{N_{3}}{N_{2}}}, \\
& \rho_{2}=\sqrt{2 h \frac{N_{7}}{N_{6}}}, \\
& \rho_{3}=\rho_{2} .
\end{aligned}
$$

The next step is to find the region of the parameters where the determinant $J_{j}$ of the Jacobian matrix, $D_{r, \alpha}\left(f_{1}, f_{2}\right)$ evaluated in the zeros $\left(r_{j}, \alpha_{j}\right)$ is non null. We find that

$$
\begin{aligned}
& J_{1}=\frac{h r_{1}^{2}}{72} N_{3} N_{4}, \\
& J_{2}=-\frac{h r_{2}^{2}}{72} N_{4} N_{7}, \\
& J_{3}=J_{2} .
\end{aligned}
$$

Now, we finish the proof of Theorem 1.3. Indeed, if $(A, B, m, \lambda, \Lambda) \in \Omega_{h}^{1} \backslash \Omega_{h}^{2}$, then $r_{1}>0, \rho_{1}>0$ and $J_{1} \neq 0$ and therefore we obtain at least one-family of periodic orbits. If $(A, B, m, \lambda, \Lambda) \in \Omega_{h}^{2} \backslash \Omega_{h}^{1}$, then $r_{2}>0, \rho_{2}>0$ and $J_{2} \neq 0$, so we get at least two-families of periodic orbits. Finally, if $(A, B, m, \lambda, \Lambda) \in \Omega_{h}^{1} \cap \Omega_{h}^{2}$, then $r_{1}>0, r_{2}>0, \rho_{1}>0, \rho_{2}>0, J_{1} \neq 0$ and $J_{2} \neq 0$, so we get at least three-families of periodic orbits. This completes the proof of Theorem 1.3.

For the proof of Theorem 1.4, observe that if we denote by $\lambda_{1}^{1}$ and $\lambda_{2}^{1}$ the eigenvalues of the Jacobian matrix $D_{r, \alpha}\left(f_{1}, f_{2}\right)$ evaluate in $\left(r_{1}, \alpha_{1}\right)$, we have that

$$
\lambda_{1}^{1}=-\frac{1}{6 \sqrt{2}} r_{1} \sqrt{h} \sqrt{-N_{3} N_{4}}, \lambda_{2}^{1}=-\lambda_{1}^{1} .
$$

Moreover, as $J_{1}=\lambda_{1}^{1} \lambda_{2}^{1}$, we obtain that $J_{1}=-\left(\lambda_{1}^{1}\right)^{2}$. Therefore, for $(A, B, m, \lambda, \Lambda) \in \Omega_{h}^{1} \backslash \Omega_{h}^{2}$ such that $N_{3} N_{4}>0$ we obtain at least one-family linearly stable and linearly unstable if $N_{3} N_{4}<0$. This proves the first part of Theorem 1.4. For the second part of Theorem 1.4, we denote by $\lambda_{1}^{2}$ and $\lambda_{2}^{2}$ the eigenvalues of the Jacobian matrix $D_{r, \alpha}\left(f_{1}, f_{2}\right)$ evaluated in $\left(r_{2}, \alpha_{2}\right)$, we have that

$$
\lambda_{1}^{2}=-\frac{1}{6 \sqrt{2}} r_{2} \sqrt{h} \sqrt{N_{4} N_{7}}, \lambda_{2}^{2}=-\lambda_{1}^{2} .
$$

Moreover, as $J_{2}=\lambda_{1}^{2} \lambda_{2}^{2}$, we obtain that $J_{2}=-\left(\lambda_{1}^{2}\right)^{2}$. Therefore, for $(A, B, m, \lambda, \Lambda) \in \Omega_{h}^{2} \backslash \Omega_{h}^{1}$ such that $N_{4} N_{7}<0$ we obtain at least two-families linearly stable and linearly unstable if $N_{4} N_{7}>0$. Finally, for the proof of the third part of Theorem 1.4, for $(A, B, m, \lambda, \Lambda) \in \Omega_{h}^{1} \cap \Omega_{h}^{2}$ such that $N_{3} N_{4}>$ 
0 and $N_{4} N_{7}<0$ we obtain three-families linearly stable and three-families linearly unstable in the other conditions. Therefore, the proof is completed.

\section{Numerical Examples on the Existence of Periodic Orbits}

\subsection{Examples of families of periodic orbits for $H^{+}$}

Taking $\lambda=\Lambda=m=0$, that is, $V_{4} \equiv 0$, we recuperate the results obtained by Jiménez-Lara and Llibre in [17]. Moreover, we emphasize that adding to the classical Henón-Heiles system a homogeneous polynomial of degree four, we obtain periodic orbits outside the region of the existence of periodic solutions found in [17]. The importance of adding to classical Henón-Heiles system a homogeneous polynomial of degree fourth, lies in to find another family of periodic orbits in a region where the conditions given by Llibre and Jiménez-Lara in [17] are not valid.

Indeed, we point out the following examples.

For a one periodic orbit: Taking $A=1, B=4, m=1.5$ and $\Lambda=-\lambda=3$, we obtain $r_{1}=$ $0.780988 \sqrt{h}, \rho_{1}=1.17901 \sqrt{h}$ and $J_{1}=552.774 h^{2}$ for every $h>0$ small. Therefore, the Hamiltonian

$$
H=\frac{1}{2}\left(p_{x}^{2}+p_{y}^{2}\right)+\frac{1}{2}\left(x^{2}+y^{2}\right)+\frac{1}{3} x^{3}+4 x y^{2}+\frac{3}{4} x^{4}+\frac{3}{4} x^{2} y^{2}-\frac{3}{4} y^{4}
$$

has at least one family of periodic orbits for every $h>0$ small. However, note that

$$
(2 B-5 A)(2 B-A)=21>0,
$$

therefore, $A$ and $B$ do not satisfy the condition given in [17].

For two periodic orbits: Taking $A=B=2, m=-3$ and $\Lambda=\lambda=-3$, we obtain $r_{2}=r_{3}=\sqrt{h}$, $\rho_{2}=\rho_{3}=\sqrt{h}$ and $J_{2}=J_{3}=\frac{49}{4} h^{2}$ for every $h>0$ small. Therefore, the Hamiltonian

$$
H=\frac{1}{2}\left(p_{x}^{2}+p_{y}^{2}\right)+\frac{1}{2}\left(x^{2}+y^{2}\right)+\frac{2}{3} x^{3}+2 x y^{2}-\frac{3}{4} x^{4}-\frac{3}{2} x^{2} y^{2}-\frac{3}{4} y^{4}
$$

has at least one family of periodic orbits for every $h>0$ small. However, note that

$$
A+B=4 \neq 0,
$$

therefore, $A$ and $B$ do not satisfy the condition given in [17].

For three periodic orbits: Taking $A=5, B=4, m=-3$ and $\Lambda=-\lambda=3$, we obtain, $r_{1}=$ $2 \sqrt{\frac{5}{19}} \sqrt{h}, r_{2}=r_{3}=\sqrt{\frac{19}{10}} \sqrt{h}, J_{1}=\frac{14490}{19} h^{2}, J_{2}=J_{3}=\frac{3059}{360} h^{2}$ for every $h>0$ small. Therefore, the Hamiltonian

$$
H=\frac{1}{2}\left(p_{x}^{2}+p_{y}^{2}\right)+\frac{1}{2}\left(x^{2}+y^{2}\right)+\frac{5}{3} x^{3}+4 x y^{2}+\frac{3}{4} x^{4}-\frac{3}{2} x^{2} y^{2}-\frac{3}{4} y^{4}
$$

has at least one family of periodic orbits for every $h>0$ small. However, note that

$$
B(2 B-5 A)=-68<0,
$$

therefore, $A$ and $B$ do not satisfy the condition given in [17]. 


\subsection{Examples of family of periodic orbits for $\mathrm{H}^{-}$}

Now, we are going to exhibit that the necessary conditions given in Theorem 1.1 are not empty, in fact, we will consider different particular elections of the parameters $A, B, m, \Lambda, \lambda$ such that the constraints are satisfied.

Firstly, if we take $A=B=4, m=1.5$ and $\Lambda=-\lambda=3$, then there exist $r_{1}=\sqrt{h}, \rho_{1}=$ $1.73205 \sqrt{h}$ and $J_{1}=123.469 h^{2}$ for every $h>0$ small, thus the Hamiltonian

$$
H=\frac{1}{2}\left(-p_{x}^{2}+p_{y}^{2}\right)+\frac{1}{2}\left(-x^{2}+y^{2}\right)+\frac{4}{3} x^{3}+4 x y^{2}+\frac{3}{4} x^{4}+\frac{3}{4} x^{2} y^{2}-\frac{3}{4} y^{4}
$$

has at least one family of periodic orbits for every $h>0$ small.

Secondly, considering $A=30, B=-10, m=-4$ and $\Lambda=\lambda=-3$, we obtain that there is $r_{2}=r_{3}=\frac{1}{3} \sqrt{\frac{2839}{129}} \sqrt{h}, \rho_{2}=\rho_{3}=\frac{1}{3} \sqrt{\frac{5161}{129}} \sqrt{h}$ and $J_{2}=J_{3}=-\frac{249085343}{2322} h^{2}$ for every $h>0$ small. Therefore, the Hamiltonian

$$
H=\frac{1}{2}\left(-p_{x}^{2}+p_{y}^{2}\right)+\frac{1}{2}\left(-x^{2}+y^{2}\right)+\frac{30}{3} x^{3}-10 x y^{2}-\frac{3}{4} x^{4}-2 x^{2} y^{2}-\frac{3}{4} y^{4}
$$

has at least two families of periodic orbits for every $h>0$ small.

Thirdly, taking $A=40, B=-10, m=-4$ and $\Lambda=\lambda=-3$, we obtain $r_{1}=\sqrt{\frac{5063}{2437}} \sqrt{h}, r_{2}=r_{3}=$ $3 \sqrt{\frac{157}{1087}} \sqrt{h}, \rho_{1}=\sqrt{\frac{9937}{2437}} \sqrt{h}, \rho_{2}=\rho_{2}=\sqrt{\frac{3587}{1087}} \sqrt{h}, J_{1}=\frac{5182036193 h^{2}}{43866}$ and $J_{2}=J_{3}=-\frac{174016131 h^{2}}{2174}$ for every $h>0$ small. Therefore, the Hamiltonian

$$
H=\frac{1}{2}\left(-p_{x}^{2}+p_{y}^{2}\right)+\frac{1}{2}\left(-x^{2}+y^{2}\right)+\frac{40}{3} x^{3}-10 x y^{2}-\frac{3}{4} x^{4}-2 x^{2} y^{2}-\frac{3}{4} y^{4}
$$

has at least three families of periodic orbits for every $h>0$ small.

Another important remark is the following which permit us to find regions for the existence of periodic orbits in a simple sense.

Remark 6.1. Let consider $m=\lambda=\Lambda$ and $A, B$ such that $m \neq-\frac{2}{3} B(A-6 B), A+B \neq 0, A-2 B \neq 0$ and $0<\frac{-B}{A-3 B}<1$, Then there exists at least a family of periodic orbits, for all $h>0$. Indeed, if $m=\lambda=\Lambda$ then

$$
r_{1}=\sqrt{2 h} \sqrt{\frac{-B}{A-3 B}}>0
$$

and

$$
d_{1}=\frac{5 h r_{1}^{2}}{36}(A-2 B)(A+B)(2 B(A-6 B)+3 m) \neq 0 .
$$

Therefore, there exists at least one family of periodic orbits.

\section{Acknowledgments}

The authors would like to acknowledge to the anonymous reviewer for his/her valuable comments and suggestions to improve the quality of this paper. 


\section{References}

[1] F. Alfaro, J. Llibre and E. Pérez-Chavela, Periodic orbits for a class of galactic potentials, Astrophysics and Space Science 344 (2013) 39-44.

[2] M. Anisiu and A. Pal, Spectral families of orbits for the Hénon-Heiles type potential, Rom. Astron. J. 9 (1999) 179-185.

[3] C. Belmonte, D. Boccaletti and G. Pucacco, Stability of axial orbits in galactic potentials, Cel. Mech. \& Dynam. Astron., 95, (2006) 101-116.

[4] D. Boccaletti and G. Puccaco, Theory of Orbits 2: perturbative and Geometrial Methods (SpringerVerlag, Heidelberg, New-York, 1999).

[5] M. Brack, Orbits with analytical Scaling Constants in Hénon-Heiles type potentials, Fundations of Phys. 31 (2001) 209-232.

[6] A. Buică and J. Llibre, Averaging methods for finding periodic orbits via Brouwer degree, Bull. Sci. Math. 128 (2004) 7-22.

[7] D. Carrasco-Olivera, M. Uribe and C. Vidal, Periodic solutions and its stability for Hamiltonians systems with polynomial Hamiltonian of degree four, Summited to publication.

[8] D. Carrasco and C. Vidal, Periodic orbits, Stability and Non-Integrability in a Generalized HénonHeiles Hamiltonian systems, Journal of Nonlinear Mathematical Physics 20 No. 1 (2013) 199-213.

[9] R. Conte, M. Musette and C. Verhoeven, Explicit integration of the Hénon-Heiles Hamiltonians, Journal of Nonlinear Mathematical Physics, 12, No 1 (2005) 212-227.

[10] K. Davies, T. Huston and M. Baranger, Calculations of periodic trajectories for the Hénon-Heiles Hamiltonian using the monodromy method, Chaos 2 (1992) 215-224.

[11] T. De Zeeuw and D. Merritt, Stellar orbits in a triaxial galaxy. I. Orbits in the plane of rotation, Astrophys. J., 267 (1983) 571-595.

[12] F. ElSabaa and H. Sherief, Periodic orbits of galactic motions, Astrophys and Space Sci. 167 (1990) 305-315.

[13] M. Falconi, E. A. Lacomba and C. Vidal The flow of classical mechanical cubic potential systems, Discrete and Continuous Dynamical Systems 11 No 4 (2004) 827-842.

[14] A. P. Fordy, The Hénon-Heiles system revisited, Physica D 52 (1991) 204-210.

[15] B. Grammaticos, B. Dorizzi and R. Padjen, Painlevé property and integrals of motion for the HenonHeiles system, Physics Letters A 89 (1982) 111-113.

[16] M. Hénon and C. Heiles, The applicability of the third integral of motion: some numerical experiments, Astron. J. 69 (1964), 73-84.

[17] L. Jiménez-Lara and J. Llibre, Periodic orbits and Hénon-Heiles systems, J. Phys. A: Math. Theor 44 (2011) 205103-14pp.

[18] J. Llibre and C. Vidal, Periodic orbits and non-integrability in a cosmological scalar field, J. Math. Phys. (2012) No 53 012702, 14 pp.

[19] A. Maciejewski, W. Radzki and S. Rybicki, Periodic trajectories near degenerate equilibria in the Hénon-Heiles and Yang-Mills Hamiltonian systems, J. Dyn. and Diff. Eq. 17 (2005) 475-488.

[20] A. Marchesiello and G. Pucacco, Relevance of the 1:1 resonance in galactic dynamics, Eur. Phys. J. Plus, 126, (2011) 104.

[21] A. Marchesiello and G. Pucacco, Resonances and bifurcations in systems with elliptical equipotentials, MNRAS, 428, (2013) 2029-2038.

[22] J. Moser, Periodic orbits near an equilibrium and a theorem of A. Weinstein, Commun. Pure Applied Math. 29 (1976) 727-747.

[23] J. Ozaki and S. Kurosaki, Periodic orbits of Hénon Heiles Hamiltonian, Prog. in Theo. Phys. 95 (1996) 519-529.

[24] G. Pucacco and K. Rosquist, (1+1)-dimensional separation of variables, Journal of Mathematical Physics, 48, (2007) 112903-112925.

[25] G. Pucacco and K. Rosquist, Nonstandard separability on the Minkowski plane, Journal of Nonlinear Mathematical Physics, 16, (2009) 421-430.

[26] D. Rod, Phatology of invariant sets in the Monkey Saddle, J. Diff. Eqn 14 (1973) 129-170. 
Periodic orbits associated to Hamiltonian functions of degree four

[27] J. A. Sanders and F. Verhulst, Averagin methods in nonlinear dynamical systems, Applied Mathematical Sciences (Springer, 1985).

[28] R. Scuflaire, Stability of axial orbits in analytical galactic potentials, Cel. Mech. \& Dynam. Astron., 61, (1995) 261-285. 